\title{
Existence of Mild Solutions for a Class of Fractional Evolution Equations with Compact Analytic Semigroup
}

\author{
He Yang \\ Department of Mathematics, Northwest Normal University, Lanzhou 730070, China \\ Correspondence should be addressed to He Yang, yanghe256@163.com \\ Received 2 May 2012; Revised 9 September 2012; Accepted 27 September 2012 \\ Academic Editor: Dumitru Băleanu \\ Copyright (C) 2012 He Yang. This is an open access article distributed under the Creative Commons \\ Attribution License, which permits unrestricted use, distribution, and reproduction in any \\ medium, provided the original work is properly cited. \\ This paper deals with the existence of mild solutions for a class of fractional evolution equations \\ with compact analytic semigroup. We prove the existence of mild solutions, assuming that the \\ nonlinear part satisfies some local growth conditions in fractional power spaces. An example is \\ also given to illustrate the applicability of abstract results.
}

\section{Introduction}

The differential equations involving fractional derivatives in time have recently been proved to be valuable tools in the modeling of many phenomena in various fields of engineering, physics, economics, and science. Numerous applications can be found in electrochemistry, control, porous media, electromagnetic, see for example, [1-5] and references therein. Hence the study of such equations has become an object of extensive study during recent years, see [6-23] and references therein.

In this paper, we consider the existence of the following fractional evolution equation:

$$
\begin{gathered}
D^{q} u(t)+A u(t)=f(t, u(t), G u(t)), \quad t \in J=[0, T], \\
u(0)=x_{0},
\end{gathered}
$$

where $D^{q}$ is the Caputo fractional derivative of order $q \in(0,1),-A$ is the infinitesimal generator of a compact analytic semigroup $S(\cdot)$ of uniformly bounded linear operators, $f$ is the nonlinear term and will be specified later, and 


$$
G u(t)=\int_{0}^{t} K(t, s) u(s) d s
$$

is a Volterra integral operator with integral kernel $K \in C\left(\Delta, R^{+}\right), \Delta=\{(t, s): 0 \leq s \leq t \leq$ $T\}, R^{+}=[0,+\infty)$. Throughout this paper, we denote by $K^{*}:=\max _{(t, s) \in \Delta} K(t, s)$

In some existing articles, the fractional differential equations were treated under the hypothesis that nonlinear term satisfies Lipschitz conditions or linear growth conditions. It is obvious that these conditions are not easy to be verified sometimes. To make the things more applicable, in this work, we will prove the existence of mild solutions for (1.1) under some new conditions. More precisely, the nonlinear term only satisfies some local growth conditions (see conditions $\left(\mathrm{H}_{1}\right)$ and $\left(\mathrm{H}_{2}\right)$ ). These conditions are much weaker than Lipschitz conditions and linear growth conditions. The main techniques used here are fractional calculus, theory of analytic semigroup, and Schauder fixed point theorem.

The rest of this paper is organized as follows. In Section 2, some preliminaries are given on the fractional power of the generator of a compact analytic semigroup and the definition of mild solutions of (1.1). In Section 3, we study the existence of mild solutions for (1.1). In Section 4, an example is given to illustrate the applicability of abstract results obtained in Section 3.

\section{Preliminaries}

In this section, we introduce some basic facts about the fractional power of the generator of a compact analytic semigroup and the fractional calculus that are used throughout this paper.

Let $X$ be a Banach space with norm $\|\cdot\|$. Throughout this paper, we assume that $-A$ : $D(A) \subset X \rightarrow X$ is the infinitesimal generator of a compact analytic semigroup $S(t)(t \geq 0)$ of uniformly bounded linear operator in $X$, that is, there exists $M \geq 1$ such that $\|S(t)\| \leq M$ for all $t \geq 0$. Without loss of generality, let $0 \in \rho(-A)$, where $\rho(-A)$ is the resolvent set of $-A$. Then for any $\alpha>0$, we can define $A^{-\alpha}$ by

$$
A^{-\alpha}:=\frac{1}{\Gamma(\alpha)} \int_{0}^{\infty} t^{(\alpha-1)} S(t) d t
$$

It follows that each $A^{-\alpha}$ is an injective continuous endomorphism of $X$. Hence we can define $A^{\alpha}$ by $A^{\alpha}:=\left(A^{-\alpha}\right)^{-1}$, which is a closed bijective linear operator in $X$. It can be shown that each $A^{\alpha}$ has dense domain and that $D\left(A^{\beta}\right) \subset D\left(A^{\alpha}\right)$ for $0 \leq \alpha \leq \beta$. Moreover, $A^{\alpha+\beta} x=A^{\alpha} A^{\beta} x=A^{\beta} A^{\alpha} x$ for every $\alpha, \beta \in \mathbb{R}$ and $x \in D\left(A^{\mu}\right)$ with $\mu:=\max \{\alpha, \beta, \alpha+\beta\}$, where $A^{0}=I$, $I$ is the identity in $X$. (For proofs of these facts, we refer to the literature [24-26]).

We denote by $X_{\alpha}$ the Banach space of $D\left(A^{\alpha}\right)$ equipped with norm $\|x\|_{\alpha}=\left\|A^{\alpha} x\right\|$ for $x \in D\left(A^{\alpha}\right)$, which is equivalent to the graph norm of $A^{\alpha}$. Then we have $X_{\beta} \hookrightarrow X_{\alpha}$ for $0 \leq \alpha \leq$ $\beta \leq 1$ (with $X_{0}=X$ ), and the embedding is continuous. Moreover, $A^{\alpha}$ has the following basic properties.

Lemma 2.1 (see [24]). $A^{\alpha}$ has the following properties.

(i) $S(t): X \rightarrow X_{\alpha}$ for each $t>0$ and $\alpha \geq 0$.

(ii) $A^{\alpha} S(t) x=S(t) A^{\alpha} x$ for each $x \in D\left(A^{\alpha}\right)$ and $t \geq 0$. 
(iii) For every $t>0, A^{\alpha} S(t)$ is bounded in $\mathrm{X}$ and there exists $M_{\alpha}>0$ such that

$$
\left\|A^{\alpha} S(t)\right\| \leq M_{\alpha} t^{-\alpha} .
$$

(iv) $A^{-\alpha}$ is a bounded linear operator for $0 \leq \alpha \leq 1$ in $X$.

In the following, we denote by $C\left(J, X_{\alpha}\right)$ the Banach space of all continuous functions from $J$ into $X_{\alpha}$ with supnorm given by $\|u\|_{C}=\sup _{t \in J}\|u(t)\|_{\alpha}$ for $u \in C\left(J, X_{\alpha}\right)$. From Lemma 2.1(iv), since $A^{-\alpha}$ is a bounded linear operator for $0 \leq \alpha \leq 1$, there exists a constant $C_{\alpha}$ such that $\left\|A^{-\alpha}\right\| \leq C_{\alpha}$ for $0 \leq \alpha \leq 1$.

For any $t \geq 0$, denote by $S_{\alpha}(t)$ the restriction of $S(t)$ to $X_{\alpha}$. From Lemma 2.1(i) and (ii), for any $x \in X_{\alpha}$, we have

$$
\begin{gathered}
\|S(t) x\|_{\alpha}=\left\|A^{\alpha} \cdot S(t) x\right\|=\left\|S(t) \cdot A^{\alpha} x\right\| \leq\|S(t)\| \cdot\left\|A^{\alpha} x\right\|=\|S(t)\| \cdot\|x\|_{\alpha \prime} \\
\left\|S(t) x-x_{\alpha}\right\|=\left\|A^{\alpha} \cdot S(t) x-A^{\alpha} x\right\|=\left\|S(t) \cdot A^{\alpha} x-A^{\alpha} x\right\| \longrightarrow 0
\end{gathered}
$$

as $t \rightarrow 0$. Therefore, $S(t)(t \geq 0)$ is a strongly continuous semigroup in $X_{\alpha}$, and $\left\|S_{\alpha}(t)\right\|_{\alpha} \leq$ $\|S(t)\|$ for all $t \geq 0$. To prove our main results, the following lemma is also needed.

Lemma 2.2 (see [27]). $S_{\alpha}(t)(t \geq 0)$ is an immediately compact semigroup in $X_{\alpha}$, and hence it is immediately norm-continuous.

Let us recall the following known definitions in fractional calculus. For more details, see $[16-20,23]$.

Definition 2.3. The fractional integral of order $\sigma>0$ with the lower limits zero for a function $f$ is defined by

$$
I^{\sigma} f(t)=\frac{1}{\Gamma(\sigma)} \int_{0}^{t}(t-s)^{\sigma-1} f(s) d s, \quad t>0,
$$

where $\Gamma$ is the gamma function.

The Riemann-Liouville fractional derivative of order $n-1<\sigma<n$ with the lower limits zero for a function $f$ can be written as

$$
{ }^{L} D^{\sigma} f(t)=\frac{1}{\Gamma(n-\sigma)} \frac{d^{n}}{d t^{n}} \int_{0}^{t}(t-s)^{n-\sigma-1} f(s) d s, \quad t>0, n \in \mathbb{N} .
$$

Also the Caputo fractional derivative of order $n-1<\sigma<n$ with the lower limits zero for a function $f \in C^{n}[0, \infty)$ can be written as

$$
D^{\sigma} f(t)=\frac{1}{\Gamma(n-\sigma)} \int_{0}^{t}(t-s)^{n-\sigma-1} f^{(n)}(s) d s, \quad t>0, n \in \mathbb{N} .
$$


Remark 2.4. (1) The Caputo derivative of a constant is equal to zero.

(2) If $f$ is an abstract function with values in $X$, then integrals which appear in Definition 2.3 are taken in Bochner's sense.

Lemma 2.5 (see [12]). A measurable function $h: J \rightarrow X$ is Bochner integrable if $\|h\|$ is Lebesgue integrable.

For $x \in X$, we define two families $\{U(t)\}_{t \geq 0}$ and $\{V(t)\}_{t \geq 0}$ of operators by

$$
U(t) x=\int_{0}^{\infty} \eta_{q}(\theta) S\left(t^{q} \theta\right) x d \theta, \quad V(t) x=q \int_{0}^{\infty} \theta \eta_{q}(\theta) S\left(t^{q} \theta\right) x d \theta, \quad 0<q<1,
$$

where

$$
\begin{array}{r}
\eta_{q}(\theta)=\frac{1}{q} \theta^{-1-(1 / q)} \rho_{q}\left(\theta^{-(1 / q)}\right), \quad \rho_{q}(\theta)=\frac{1}{\pi} \sum_{n=1}^{\infty}(-1)^{n-1} \theta^{-q n-1} \frac{\Gamma(n q+1)}{n !} \sin (n \pi q), \\
\theta \in(0, \infty),
\end{array}
$$

$\eta_{q}$ is a probability density function defined on $(0, \infty)$, which has properties $\eta_{q}(\theta) \geq 0$ for all $\theta \in(0, \infty)$ and

$$
\int_{0}^{\infty} \eta_{q}(\theta) d \theta=1, \quad \int_{0}^{\infty} \theta \eta_{q}(\theta) d \theta=\frac{1}{\Gamma(q+1)}
$$

The following lemma follows from the results in [7, 11-13].

Lemma 2.6. The operators $U$ and $V$ have the following properties.

(i) For fixed $t \geq 0$ and any $x \in X_{\alpha}$, we have

$$
\|U(t) x\|_{\alpha} \leq M\|x\|_{\alpha}, \quad\|V(t) x\|_{\alpha} \leq \frac{q M}{\Gamma(1+q)}\|x\|_{\alpha}=\frac{M}{\Gamma(q)}\|x\|_{\alpha} .
$$

(ii) The operators $U(t)$ and $V(t)$ are strongly continuous for all $t \geq 0$.

(iii) $U(t)$ and $V(t)$ are norm-continuous in $X$ for $t>0$.

(iv) $U(t)$ and $V(t)$ are compact operators in $X$ for $t>0$.

(v) For every $t>0$, the restriction of $U(t)$ to $X_{\alpha}$ and the restriction of $V(t)$ to $X_{\alpha}$ are normcontinuous.

(vi) For every $t>0$, the restriction of $U(t)$ to $X_{\alpha}$ and the restriction of $V(t)$ to $X_{\alpha}$ are compact operators in $X_{\alpha}$.

Based on an overall observation of the previous related literature, in this paper, we adopt the following definition of mild solution of (1.1). 
Definition 2.7. By a mild solution of (1.1), we mean a function $u \in C\left(J, X_{\alpha}\right)$ satisfying

$$
u(t)=U(t) x_{0}+\int_{0}^{t}(t-s)^{q-1} V(t-s) f(s, u(s), G u(s)) d s
$$

for all $t \in J$.

\section{Existence of Mild Solutions}

In this section, we give the existence theorems of mild solutions of (1.1). The discussions are based on fractional calculus and Schauder fixed point theorem. Our main results are as follows.

Theorem 3.1. Assume that the following condition on $f$ is satisfied.

$\left(\mathrm{H}_{1}\right)$ There exists a constant $\beta \in[\alpha, 1]$ such that $f: J \times X_{\alpha} \times X_{\alpha} \rightarrow X_{\beta}$ satisfies:

(i) for each $(x, y) \in X_{\alpha} \times X_{\alpha}$, the function $f(\cdot, x, y): J \rightarrow X_{\beta}$ is measurable;

(ii) for each $t \in J$, the function $f(t, \cdot, \cdot): X_{\alpha} \times X_{\alpha} \rightarrow X_{\beta}$ is continuous;

(iii) for any $r>0$, there exists a function $g_{r} \in L^{\infty}\left(J, \mathbb{R}^{+}\right)$such that

$$
\sup _{\|x\|_{\alpha} \leq r,\|y\|_{\alpha} \leq K^{*} T r}\|f(t, x, y)\|_{\beta} \leq g_{r}(t), \quad t \in J
$$

and there is a constant $\gamma>0$ such that

$$
\liminf _{r \rightarrow+\infty} \frac{1}{r} \int_{0}^{t} \frac{g_{r}(s)}{(t-s)^{1-q}} d s \leq r<+\infty
$$

If $x_{0} \in X_{\alpha}$ and $M C_{\beta-\alpha} \gamma<\Gamma(q)$, then (1.1) has at least one mild solution.

Proof. Define an operator $Q$ by

$$
(Q u)(t)=U(t) x_{0}+\int_{0}^{t}(t-s)^{q-1} V(t-s) f(s, u(s), G u(s)) d s, \quad t \in J .
$$

It is not difficult to verify that $Q: C\left(J, X_{\alpha}\right) \rightarrow C\left(J, X_{\alpha}\right)$. We will use Schauder fixed point theorem to prove that $Q$ has fixed points in $C\left(J, X_{\alpha}\right)$.

For any $r>0$, let $B_{r}:=\left\{u \in C\left(J, X_{\alpha}\right):\|u(t)\|_{\alpha} \leq r, t \in J\right\}$. We first show that there is a positive number $r$ such that $Q\left(B_{r}\right) \subset B_{r}$. If this were not the case, then for each $r>0$, 
there would exist $u_{r} \in B_{r}$ and $t_{r} \in J$ such that $\left\|\left(Q u_{r}\right)\left(t_{r}\right)\right\|_{\alpha}>r$. Thus, from Lemma 2.6(i) and $\left(\mathrm{H}_{1}\right)(\mathrm{iii})$, we see that

$$
\begin{aligned}
r<\left\|\left(Q u_{r}\right)\left(t_{r}\right)\right\|_{\alpha} & \leq\left\|U\left(t_{r}\right) x_{0}\right\|_{\alpha}+\int_{0}^{t_{r}}\left(t_{r}-s\right)^{q-1}\left\|V\left(t_{r}-s\right) f\left(s, u_{r}(s), G u_{r}(s)\right)\right\|_{\alpha} d s \\
& \leq M\left\|x_{0}\right\|_{\alpha}+\int_{0}^{t_{r}}\left(t_{r}-s\right)^{q-1}\left\|A^{\alpha-\beta} V\left(t_{r}-s\right) \cdot A^{\beta} f\left(s, u_{r}(s), G u_{r}(s)\right)\right\| d s \\
& \leq M\left\|x_{0}\right\|_{\alpha}+\frac{M C_{\beta-\alpha}}{\Gamma(q)} \int_{0}^{t_{r}}\left(t_{r}-s\right)^{q-1} g_{r}(s) d s
\end{aligned}
$$

Dividing on both sides by $r$ and taking the lower limit as $r \rightarrow+\infty$, we have

$$
M C_{\beta-\alpha} \gamma \geq \Gamma(q)
$$

which is a contradiction. Hence $Q\left(B_{r}\right) \subset B_{r}$ for some $r>0$.

To complete the proof, we separate the rest of proof into the following three steps.

Step 1. $Q: B_{r} \rightarrow B_{r}$ is continuous.

Let $\left\{u_{n}\right\} \subset B_{r}$ with $u_{n} \rightarrow u \in B_{r}$ as $n \rightarrow \infty$. From the assumption $\left(\mathrm{H}_{1}\right)$ (ii), for each $s \in J$, we have

$$
f\left(s, u_{n}(s), G u_{n}(s)\right) \longrightarrow f(s, u(s), G u(s))
$$

as $n \rightarrow \infty$. Since $\left\|f\left(s, u_{n}(s), G u_{n}(s)\right)-f(s, u(s), G u(s))\right\|_{\beta} \leq 2 g_{r}(s)$, by the Lebesgue dominated convergence theorem, for each $t \in J$, we have

$$
\begin{aligned}
&\left\|\left(Q u_{n}\right)(t)-(Q u)(t)\right\|_{\alpha} \leq \int_{0}^{t}(t-s)^{q-1}\left\|V(t-s)\left[f\left(s, u_{n}(s), G u_{n}(s)\right)-f(s, u(s), G u(s))\right]\right\|_{\alpha} d s \\
& \leq \int_{0}^{t}(t-s)^{q-1} \| A^{\alpha-\beta} V(t-s) \\
& \leq \frac{M C_{\beta-\alpha}}{\Gamma(q)} \int_{0}^{t}(t-s)^{q-1} \| f\left(s, u_{n}(s), G u_{n}(s)\right) \\
&-f(s, u(s), G u(s)) \|_{\beta} d s \rightarrow 0
\end{aligned}
$$

as $n \rightarrow \infty$, which implies that $Q: B_{r} \rightarrow B_{r}$ is continuous. 
Step 2. $\left(Q B_{r}\right)(t):=\left\{(Q u)(t): u \in B_{r}\right\}$ is relatively compact in $X_{\alpha}$ for all $t \in J$.

It follows from (2.9) and (3.3) that $\left(Q B_{r}\right)(0)=\left\{(Q u)(0): u \in B_{r}\right\}=\left\{x_{0}\right\}$ is compact in $X_{\alpha}$. Hence it is only necessary to consider the case of $t>0$. For each $t \in(0, T], \epsilon \in(0, t)$, and any $\delta>0$, we define a set $\left(Q_{\epsilon, \delta} B_{r}\right)(t)$ by

$$
\left(Q_{e, \delta} B_{r}\right)(t):=\left\{\left(Q_{\epsilon, \delta} u\right)(t): u \in B_{r}\right\},
$$

where

$$
\begin{aligned}
\left(Q_{\epsilon, \delta} u\right)(t)= & \int_{\delta}^{\infty} \eta_{q}(\theta) S\left(t^{q} \theta\right) d \theta x_{0} \\
& +q \int_{0}^{t-\epsilon}(t-s)^{q-1} \int_{\delta}^{\infty} \theta \eta_{q}(\theta) S\left((t-s)^{q} \theta\right) d \theta \cdot f(s, u(s), G u(s)) d s \\
= & S\left(\epsilon^{q} \delta\right)\left[\int_{\delta}^{\infty} \eta_{q}(\theta) S\left(t^{q} \theta-\epsilon^{q} \delta\right) d \theta x_{0}\right. \\
& \left.+q \int_{0}^{t-\epsilon}(t-s)^{q-1} \int_{\delta}^{\infty} \theta \eta_{q}(\theta) S\left((t-s)^{q} \theta-\epsilon^{q} \delta\right) d \theta \cdot f(s, u(s), G u(s)) d s\right] .
\end{aligned}
$$

Then the set $\left(Q_{\epsilon, \delta} B_{r}\right)(t)$ is relatively compact in $X_{\alpha}$ since by Lemma 2.2, the operator $S_{\alpha}\left(\epsilon^{q} \delta\right)$ is compact in $X_{\alpha}$. For any $u \in B_{r}$ and $t \in(0, T]$, from the following inequality:

$$
\begin{aligned}
\left\|(Q u)(t)-\left(Q_{\epsilon, \delta} u\right)(t)\right\|_{\alpha} \leq & \left\|\int_{0}^{\delta} \eta_{q}(\theta) S\left(t^{q} \theta\right) d \theta x_{0}\right\|_{\alpha} \\
+ & \| q \int_{0}^{t}(t-s)^{q-1} \int_{0}^{\delta} \theta \eta_{q}(\theta) S\left((t-s)^{q} \theta\right) d \theta \\
& \cdot f(s, u(s), G u(s)) d s \|_{\alpha} \\
+ & \| q \int_{0}^{t}(t-s)^{q-1} \int_{\delta}^{\infty} \theta \eta_{q}(\theta) S\left((t-s)^{q} \theta\right) d \theta \cdot f(s, u(s), G u(s)) d s \\
& -q \int_{0}^{t-\epsilon}(t-s)^{q-1} \int_{\delta}^{\infty} \theta \eta_{q}(\theta) S\left((t-s)^{q} \theta\right) d \theta \\
& \cdot f(s, u(s), G u(s)) d s \|_{\alpha} \\
& M\left\|x_{0}\right\|_{\alpha} \int_{0}^{\delta} \eta_{q}(\theta) d \theta+q M C_{\beta-\alpha}\left\|g_{r}\right\|_{L^{\infty}} \int_{0}^{t}(t-s)^{q-1} d s \int_{0}^{\delta} \theta \eta_{q}(\theta) d \theta \\
+ & q M C_{\beta-\alpha}\left\|g_{r}\right\|_{L^{\infty}} \int_{t-\epsilon}^{t}(t-s)^{q-1} d s \int_{0}^{\infty} \theta \eta_{q}(\theta) d \theta
\end{aligned}
$$




$$
\begin{gathered}
\leq M\left\|x_{0}\right\|_{\alpha} \int_{0}^{\delta} \eta_{q}(\theta) d \theta+M C_{\beta-\alpha} T^{q}\left\|g_{r}\right\|_{L^{\infty}} \\
\times \int_{0}^{\delta} \theta \eta_{q}(\theta) d \theta+\frac{M C_{\beta-\alpha}\left\|g_{r}\right\|_{L^{\infty}}}{\Gamma(q+1)} \epsilon^{q} .
\end{gathered}
$$

One can obtain that the set $\left(Q B_{r}\right)(t)$ is relatively compact in $X_{\alpha}$ for all $t \in(0, T)$. And since it is compact at $t=0$, we have the relatively compactness of $\left(Q B_{r}\right)(t)$ in $X_{\alpha}$ for all $t \in J$.

Step 3. $Q B_{r}:=\left\{Q u \in C\left(J, X_{\alpha}\right): u \in B_{r}\right\}$ is equicontinuous.

For $\tau \in[0, T)$, by (3.3), we have

$$
\begin{aligned}
\|(Q u)(\tau)-(Q u)(0)\|_{\alpha} \leq & \left\|U(\tau) x_{0}-x_{0}\right\|_{\alpha} \\
& +\left\|\int_{0}^{\tau}(\tau-s)^{q-1} V(\tau-s) f(s, u(s), G u(s)) d s\right\|_{\alpha} \\
\leq & \|U(\tau)-I\| \cdot\left\|x_{0}\right\|_{\alpha}+\frac{M C_{\beta-\alpha}\left\|g_{r}\right\|_{L^{\infty}}}{\Gamma(q+1)} \tau^{q} .
\end{aligned}
$$

Hence it is only necessary to consider the case of $t>0$. For $0<t_{1}<t_{2} \leq T$, by Lemma 2.1 and Lemma 2.6(i), we have

$$
\begin{aligned}
\left\|(Q u)\left(t_{2}\right)-(Q u)\left(t_{1}\right)\right\|_{\alpha} \leq & \left\|U\left(t_{2}\right) x_{0}-U\left(t_{1}\right) x_{0}\right\|_{\alpha} \\
& +\| \int_{0}^{t_{2}}\left(t_{2}-s\right)^{q-1} V\left(t_{2}-s\right) f(s, u(s), G u(s)) d s \\
& \quad-\int_{0}^{t_{1}}\left(t_{1}-s\right)^{q-1} V\left(t_{1}-s\right) f(s, u(s), G u(s)) d s \|_{\alpha} \\
\leq & \left\|U\left(t_{2}\right)-U\left(t_{1}\right)\right\| \cdot\left\|x_{0}\right\|_{\alpha} \\
& +\left\|\int_{t_{1}}^{t_{2}}\left(t_{2}-s\right)^{q-1} V\left(t_{2}-s\right) f(s, u(s), G u(s)) d s\right\|_{\alpha} \\
& +\left\|\int_{0}^{t_{1}}\left[\left(t_{2}-s\right)^{q-1}-\left(t_{1}-s\right)^{q-1}\right] V\left(t_{2}-s\right) f(s, u(s), G u(s)) d s\right\|_{\alpha} \\
& +\left\|\int_{0}^{t_{1}}\left(t_{1}-s\right)^{q-1} f(s, u(s), G u(s))\left[V\left(t_{2}-s\right)-V\left(t_{1}-s\right)\right] d s\right\|_{\alpha} \\
\leq & \left\|U\left(t_{2}\right)-U\left(t_{1}\right)\right\| \cdot\left\|x_{0}\right\|_{\alpha}+\frac{M C_{\beta-\alpha}\left\|g_{r}\right\|_{L^{\infty}}}{\Gamma\left(t_{2}-t_{1}\right)^{q}} \\
& +\frac{M C_{\beta-\alpha}\left\|g_{r}\right\|_{L^{\infty}}}{\Gamma(q+1)}\left[t_{2}^{q}-t_{1}^{q}-\left(t_{2}-t_{1}\right)^{q}\right]
\end{aligned}
$$




$$
\begin{aligned}
& \quad+\left\|\int_{0}^{t_{1}}\left(t_{1}-s\right)^{q-1}\left[V\left(t_{2}-s\right)-V\left(t_{1}-s\right)\right] \cdot f(s, u(s), G u(s)) d s\right\|_{\alpha} \\
& \triangleq I_{1}+I_{2}+I_{3}+I_{4} .
\end{aligned}
$$

From Lemma 2.6(v), we see that $I_{1} \rightarrow 0$ as $t_{2} \rightarrow t_{1}$ independently of $u \in B_{r}$. From the expressions of $I_{2}$ and $I_{3}$, it is clear that $I_{2} \rightarrow 0$ and $I_{3} \rightarrow 0$ as $t_{2} \rightarrow t_{1}$ independently of $u \in B_{r}$. For any $\epsilon \in\left(0, t_{1}\right)$, we have

$$
\begin{aligned}
I_{4} \leq & \left\|\int_{0}^{t_{1}-\epsilon}\left(t_{1}-s\right)^{q-1}\left[V\left(t_{2}-s\right)-V\left(t_{1}-s\right)\right] \cdot f(s, u(s), G u(s)) d s\right\|_{\alpha} \\
& +\left\|\int_{t_{1}-\epsilon}^{t_{1}}\left(t_{1}-s\right)^{q-1}\left[V\left(t_{2}-s\right)-V\left(t_{1}-s\right)\right] \cdot f(s, u(s), G u(s)) d s\right\|_{\alpha} \\
& \leq \frac{1}{q} C_{\beta-\alpha}\left\|g_{r}\right\|_{L^{\infty}}\left(T^{q}+\epsilon^{q}\right) \sup _{0 \leq s \leq t_{1}-\epsilon}\left\|V\left(t_{2}-s\right)-V\left(t_{1}-s\right)\right\|+\frac{2 M C_{\beta-\alpha}\left\|g_{r}\right\|_{L^{\infty}}}{\Gamma(q+1)} \epsilon^{q} .
\end{aligned}
$$

It follows from Lemma 2.6(v) that $I_{4} \rightarrow 0$ as $t_{2} \rightarrow t_{1}$ and $\epsilon \rightarrow 0$ independently of $u \in B_{r}$. Therefore, we prove that $Q B_{r}$ is equicontinuous.

Thus, the Arzela-Ascoli theorem guarantees that $Q$ is a compact operator. By the Schauder fixed point theorem, the operator $Q$ has at least one fixed point $u^{*}$ in $B_{r}$, which is a mild solution of (1.1). This completes the proof.

Remark 3.2. In assumption $\left(\mathrm{H}_{1}\right)$ (iii), if the function $g_{r}(t)$ is independent of $t$, then we can easily obtain a constant $\gamma>0$ satisfying (3.2). For example, if there is a constant $a_{f}>0$ such that

$$
\|f(t, x, y)\|_{\beta} \leq a_{f}\left(1+\|x\|_{\alpha}+\|y\|_{\alpha}\right)
$$

for all $x, y \in X_{\alpha}$ and $t \in J$, then for any $r>0, x, y \in X_{\alpha}$ with $\|x\|_{\alpha} \leq r,\|y\|_{\alpha} \leq K^{*} T r$, we have $\|f(t, x, y)\|_{\beta} \leq a_{f}+a_{f}\left(1+K^{*} T\right) r \triangleq g_{r}(t)$, where $g_{r}(t)$ is independent of $t$. Thus, $\gamma:=(1 / q) a_{f} T^{q}\left(1+K^{*} T\right)>0$ is the constant in (3.2).

More generally, if $f$ satisfies the following condition:

(H2) there is a constant $\beta \in[\alpha, 1]$ such that $f: J \times X_{\alpha} \times X_{\alpha} \rightarrow X_{\beta}$ satisfies:

(i) for each $(x, y) \in X_{\alpha} \times X_{\alpha}$, the function $f(\cdot, x, y): J \rightarrow X_{\beta}$ is measurable,

(ii) for any $r>0$, there exists a function $\ell \in L^{\infty}\left(J, \mathbb{R}^{+}\right)$such that

$$
\left\|f\left(t, x_{1}, y_{1}\right)-f\left(t, x_{2}, y_{2}\right)\right\|_{\beta} \leq \ell(t)\left(\left\|x_{1}-x_{2}\right\|_{\alpha}+\left\|y_{1}-y_{2}\right\|_{\alpha}\right)
$$

for any $x_{i}, y_{i} \in X_{\alpha}$ with $\left\|x_{i}\right\|_{\alpha} \leq r,\left\|y_{i}\right\|_{\alpha} \leq K^{*} \operatorname{Tr}(i=1,2)$ and $t \in J$, then we have the following existence and uniqueness theorem. 
Theorem 3.3. Assume that the condition $\left(H_{2}\right)$ is satisfied. If $x_{0} \in X_{\alpha}$ and $M C_{\beta-\alpha} T^{q}(1+$ $\left.K^{*} T\right)\|\ell\|_{L^{\infty}}<\Gamma(q+1)$, then (1.1) has a unique mild solution.

Proof. For any $r>0$, if $x, y \in X_{\alpha}$ with $\|x\|_{\alpha} \leq r,\|y\|_{\alpha} \leq K^{*} T r$, then from $\left(\mathrm{H}_{2}\right)$ (ii), we have

$$
\|f(t, x, y)\|_{\beta} \leq \ell(t)\left(1+K^{*} T\right) r+b(t) \triangleq g_{r}(t)
$$

where $b(t)=\|f(t, 0,0)\|_{\beta}$. Therefore, the condition $\left(\mathrm{H}_{1}\right)$ (iii) is satisfied with $\gamma=((1+$ $\left.\left.K^{*} T\right) T^{q}\|\ell\|_{L^{\infty}}\right) / q$. By Theorem 3.1, (1.1) has at least one mild solution $u^{*} \in B_{r}$.

Let $u_{1}, u_{2} \in B_{r}$ be the solutions of (1.1). We show that $u_{1} \equiv u_{2}$. Since $u_{1}(t)=\left(Q u_{1}\right)(t)$ and $u_{2}(t)=\left(Q u_{2}\right)(t)$ for all $t \in J$, we have

$$
\begin{aligned}
\left\|u_{1}(t)-u_{2}(t)\right\|_{\alpha} & =\left\|\left(Q u_{1}\right)(t)-\left(Q u_{2}\right)(t)\right\|_{\alpha} \\
& \leq \int_{0}^{t}(t-s)^{q-1}\left\|V(t-s)\left[f\left(s, u_{1}(s), G u_{1}(s)\right)-f\left(s, u_{2}(s), G u_{2}(s)\right)\right]\right\|_{\alpha} d s \\
& =\int_{0}^{t}(t-s)^{q-1}\left\|A^{\alpha-\beta} V(t-s) \cdot A^{\beta}\left[f\left(s, u_{1}(s), G u_{1}(s)\right)-f\left(s, u_{2}(s), G u_{2}(s)\right)\right]\right\| d s \\
& \leq \frac{M C_{\beta-\alpha}}{\Gamma(q)} \int_{0}^{t}(t-s)^{q-1} \cdot\left\|f\left(s, u_{1}(s), G u_{1}(s)\right)-f\left(s, u_{2}(s), G u_{2}(s)\right)\right\|_{\beta} d s \\
& \leq \frac{M C_{\beta-\alpha}}{\Gamma(q)} \int_{0}^{t}(t-s)^{q-1} \cdot \ell(s)\left(\left\|u_{1}(s)-u_{2}(s)\right\|_{\alpha}+\left\|G u_{1}(s)-G u_{2}(s)\right\|_{\alpha}\right) d s \\
& \leq \frac{M C_{\beta-\alpha}\|\ell\|_{L^{\infty}}}{\Gamma(q)}\left(1+K^{*} T\right) \int_{0}^{t}(t-s)^{q-1} \cdot\left\|u_{1}(s)-u_{2}(s)\right\|_{\alpha} d s .
\end{aligned}
$$

By using the Gronwall-Bellman inequality (see [14, Theorem 1]), we can deduce that $\| u_{1}(t)-$ $u_{2}(t) \|_{\alpha}=0$ for all $t \in J$, which implies that $u_{1} \equiv u_{2}$. Hence (1.1) has a unique mild solution $u^{*} \in B_{r}$. This completes the proof.

Remark 3.4. In Theorem 3.3, we only assume that $f$ satisfies a local Lioschitz condition (see condition $\left.\left(\mathrm{H}_{2}\right)\right)$, and an existence and uniqueness result is obtained. If $f(t, u, v) \equiv f(t, u)$ : $J \times X_{\alpha} \rightarrow X$, then the assumption $\left(\mathrm{H}_{2}\right)$ deletes the linear growth condition (3) of assumption (Hf) in [12]. Therefore, the Theorem 3.3 extends and improves the main result in [12].

\section{An Example}

Assume that $X=L^{2}[0, \pi]$ equipped with its natural norm and inner product defined, respectively, for all $u, v \in L^{2}[0, \pi]$, by

$$
\|u\|_{X}=\left(\int_{0}^{\pi}|u(x)|^{2} d x\right)^{1 / 2}, \quad\langle u, v\rangle=\int_{0}^{\pi} u(x) \overline{v(x)} d x
$$


Consider the following fractional partial differential equation:

$$
\begin{aligned}
\frac{\partial^{1 / 2}}{\partial t^{1 / 2}} u(x, t)-\frac{\partial^{2}}{\partial x^{2}} u(x, t)= & g\left(x, t, u(x, t), \int_{0}^{t} K(t, s) u(x, s) d s\right), \quad t \in[0, T], x \in[0, \pi] \\
& u(0, t)=u(\pi, t)=0, \quad t \in[0, T] \\
& u(x, 0)=u_{0}(x), \quad x \in[0, \pi]
\end{aligned}
$$

where $T>0$ is a constant.

Let the operator $A: D(A) \subset X \rightarrow X$ be defined by

$$
D(A):=\left\{v \in X: v^{\prime \prime} \in X, v(0)=v(\pi)=0\right\}, \quad A u=-\frac{\partial^{2} u}{\partial x^{2}} .
$$

It is well known that $A$ has a discrete spectrum with eigenvalues of the form $n^{2}, n \in \mathbb{N}$, and corresponding normalized eigenfunctions given by $z_{n}=\sqrt{(2 / \pi)} \sin (n x)$. Moreover, $-A$ generates a compact analytic semigroup $S(t)(t \geq 0)$ in $\mathrm{X}$, and

$$
S(t) u=\sum_{n=1}^{\infty} e^{-n^{2} t}\left\langle u, z_{n}\right\rangle z_{n}
$$

It is not difficult to verify that $\|S(t)\|_{\mathcal{L}(X)} \leq e^{-t}$ for all $t \geq 0$. Hence, we take $M=1$.

The following results are also well known.

(I) The operator $A$ can be written as

$$
A u=\sum_{n=1}^{\infty} n^{2}\left\langle u, z_{n}\right\rangle z_{n}
$$

for every $u \in D(A)$.

(II) The operator $A^{1 / 2}$ is given by

$$
A^{1 / 2} u=\sum_{n=1}^{\infty} n\left\langle u, z_{n}\right\rangle z_{n}
$$

for each $u \in D\left(A^{1 / 2}\right):=\left\{v \in X: \sum_{n=1}^{\infty} n<v, z_{n}>z_{n} \in X\right\}$ and $\left\|A^{-(1 / 2)}\right\|_{\mathcal{L}(X)}=1$.

Lemma 4.1 (see [28]). If $m \in D\left(A^{1 / 2}\right)$, then $m$ is absolutely continuous, $m^{\prime} \in X$ and $\left\|m^{\prime}\right\|_{X}=$ $\left\|A^{1 / 2} m\right\|_{\mathrm{X}}$.

Let $X_{1 / 2}=\left(D\left(A^{1 / 2}\right),\|\cdot\|_{1 / 2}\right)$, where $\|x\|_{1 / 2}:=\left\|A^{1 / 2} x\right\|_{X}$ for all $x \in D\left(A^{1 / 2}\right)$. Assume that $g:[0, \pi] \times[0, T] \times \mathbb{R} \times \mathbb{R} \rightarrow \mathbb{R}$ satisfies the following conditions.

(i) For each $(x, t) \in[0, \pi] \times[0, T]$, the function $g(x, t, \cdot, \cdot)$ is continuous.

(ii) For each $(\xi, \eta) \in \mathbb{R}^{2}$, the function $g(\cdot, \cdot, \xi, \eta)$ is measurable. 
(iii) For each $t \in[0, T]$ and $\xi, \eta \in \mathbb{R}, g(\cdot, t, \xi, \eta)$ is differentiable, and $(\partial / \partial x) g(x, t, \xi, \eta) \in$ $X$.

(iv) $g(0, \cdot, \cdot, \cdot)=g(\pi, \cdot, \cdot, \cdot)=0$.

(v) There exist the functions $\ell_{1}, \ell_{0} \in L^{\infty}\left([0, T], \mathbb{R}^{+}\right)$such that

$$
\left|\frac{\partial}{\partial x} g(x, t, \xi, \eta)\right| \leq \ell_{1}(t)(|\xi|+|\eta|)+\ell_{0}(t)
$$

for all $(x, t, \xi, \eta) \in[0, \pi] \times[0, T] \times \mathbb{R} \times \mathbb{R}$.

Define $f(t, u(t), G u(t))(x)=g\left(x, t, u(x, t), \int_{0}^{t} K(t, s) u(x, s) d s\right)$. Then, for each $\phi \in X_{1 / 2}$, from assumptions (iii) and (iv), we have

$$
\begin{aligned}
\left\langle f(t, \phi, G \phi), z_{n}\right\rangle & =\int_{0}^{\pi} g\left(x, t, \phi(x, t), \int_{0}^{t} K(t, s) \phi(x, s) d s\right) \cdot \sqrt{\frac{2}{\pi}} \sin (n x) d x \\
& =\frac{1}{n} \int_{0}^{\pi}\left(\frac{\partial}{\partial x} g\left(x, t, \phi(x, t), \int_{0}^{t} K(t, s) \phi(x, s) d s\right)\right) \cdot \sqrt{\frac{2}{\pi}} \cos (n x) d x .
\end{aligned}
$$

This implies from (II) that $f:[0, T] \times X_{1 / 2} \times X_{1 / 2} \rightarrow X_{1 / 2}$. Moreover, for any $r>0$, by Minkowski inequality, assumption (v) and Lemma 4.1, we have

$$
\begin{aligned}
\sup _{\|\phi\|_{1 / 2} \leq r}\|f(t, \phi, G \phi)\|_{1 / 2} & =\sup _{\|\phi\|_{1 / 2} \leq r}\left\|\frac{\partial}{\partial x} g\left(x, t, \phi(x, t), \int_{0}^{t} K(t, s) \phi(x, s) d s\right)\right\|_{X} \\
& =\sup _{\|\phi\|_{1 / 2} \leq r}\left(\int_{0}^{\pi}\left|\frac{\partial}{\partial x} g\left(x, t, \phi(x, t), \int_{0}^{t} K(t, s) \phi(x, s) d s\right)\right|^{2} d x\right)^{1 / 2} \\
& \leq \sup _{\|\phi\|_{1 / 2} \leq r}\left(\int _ { 0 } ^ { \pi } \left[\ell_{1}(t)\left(|\phi(x, t)|+\left|\int_{0}^{t} K(t, s) \phi(x, s) d s\right|\right)\right.\right. \\
& \leq \sup _{\|\phi\|_{1 / 2} \leq r}\left[\ell_{1}(t)\left(\|\phi\|_{X}+K^{*} T\|\phi\|_{X}\right)+\ell_{0}(t)\right] \\
& =\sup _{\|\phi\|_{1 / 2} \leq r}\left[\left(1+K^{*} T\right) \ell_{1}(t)\left\|A^{-(1 / 2)} \cdot A^{1 / 2} \phi\right\|_{X}+\ell_{0}(t)\right] \\
& \leq\left(1+K^{*} T\right) r \ell_{1}(t)+\ell_{0}(t) \triangleq g_{r}(t) .
\end{aligned}
$$

Therefore, $f$ satisfies the condition $\left(\mathrm{H}_{1}\right)$ with $\gamma=2 T^{1 / 2}\left(1+K^{*} T\right)\left\|\ell_{1}\right\|_{\left(L^{\infty}\right)}$. Thus, (4.2) has at least one mild solution provided that $\gamma<\sqrt{\pi}$ due to Theorem 3.1. 
Abstract and Applied Analysis

Assume furthermore that the function $g$ satisfies the following:

(vi) for any $r>0$, there exists a function $\ell_{2} \in L^{\infty}\left([0, T], \mathbb{R}^{+}\right)$such that

$$
\left|\frac{\partial}{\partial x} g\left(x, t, \xi_{1}, \eta_{1}\right)-\frac{\partial}{\partial x} g\left(x, t, \xi_{2}, \eta_{2}\right)\right| \leq \ell_{2}(t)\left(\left|\xi_{1}-\xi_{2}\right|+\left|\eta_{1}-\eta_{2}\right|\right)
$$

for $\left(x, t, \xi_{1}, \eta_{1}\right),\left(x, t, \xi_{2}, \eta_{2}\right) \in[0, \pi] \times[0, T] \times \mathbb{R} \times \mathbb{R}$ with $\left|\xi_{i}\right| \leq r$ and $\left|\eta_{i}\right| \leq K^{*} T r, i=1,2$.

Then for each $\phi_{1}, \phi_{2} \in X_{1 / 2}$, by Lemma 4.1 , we have

$$
\begin{aligned}
& \left\|f\left(t, \phi_{1}, G \phi_{1}\right)-f\left(t, \phi_{2}, G \phi_{2}\right)\right\|_{1 / 2}=\left\|A^{1 / 2}\left[f\left(t, \phi_{1}, G \phi_{1}\right)-f\left(t, \phi_{2}, G \phi_{2}\right)\right]\right\|_{X} \\
& =\| \frac{\partial}{\partial x} g\left(x, t, \phi_{1}(x, t), \int_{0}^{t} K(t, s) \phi_{1}(x, s) d s\right) \\
& -\frac{\partial}{\partial x} g\left(x, t, \phi_{2}(x, t), \int_{0}^{t} K(t, s) \phi_{2}(x, s) d s\right) \|_{X} \\
& =\left(\int_{0}^{\pi} \frac{\partial}{\partial x} g\left(x, t, \phi_{1}(x, t), \int_{0}^{t} K(t, s) \phi_{1}(x, s) d s\right)\right. \\
& \left.-\left.\frac{\partial}{\partial x} g\left(x, t, \phi_{2}(x, t), \int_{0}^{t} K(t, s) \phi_{2}(x, s) d s\right)\right|^{2} d x\right)^{1 / 2} \\
& \leq\left(\int_{0}^{\pi} \mid \ell_{2}(t)\left(\left|\phi_{1}(x, t)-\phi_{2}(x, t)\right|\right.\right. \\
& +\mid \int_{0}^{t} K(t, s) \phi_{1}(x, s) d s \\
& \left.\left.-\int_{0}^{t} K(t, s) \phi_{2}(x, s) d s \mid\right)\left.\right|^{2} d x\right)^{1 / 2} \\
& \leq \ell_{2}(t)\left[\left(\int_{0}^{\pi}\left|\phi_{1}(x, t)-\phi_{2}(x, t)\right|^{2} d x\right)^{1 / 2}\right. \\
& +\left(\int_{0}^{\pi} \mid \int_{0}^{t} K(t, s) \phi_{1}(x, s) d s\right. \\
& \left.\left.-\left.\int_{0}^{t} K(t, s) \phi_{2}(x, s) d s\right|^{2} d x\right)^{1 / 2}\right]
\end{aligned}
$$




$$
\begin{aligned}
& =\ell_{2}(t)\left(\left\|\phi_{1}-\phi_{2}\right\|_{X}+\left\|G \phi_{1}-G \phi_{2}\right\|_{X}\right) \\
& =\ell_{2}(t)\left(\left\|A^{-(1 / 2)} \cdot A^{1 / 2}\left(\phi_{1}-\phi_{2}\right)\right\|_{X}\right. \\
& \left.\quad+\left\|A^{-(1 / 2)} \cdot A^{1 / 2}\left(G \phi_{1}-G \phi_{2}\right)\right\|_{X}\right) \\
& \quad \leq \ell_{2}(t)\left(\left\|A^{1 / 2}\left(\phi_{1}-\phi_{2}\right)\right\|_{X}+\left\|A^{1 / 2}\left(G \phi_{1}-G \phi_{2}\right)\right\|_{X}\right) \\
& =\ell_{2}(t)\left(\left\|\phi_{1}-\phi_{2}\right\|_{1 / 2}+\left\|G \phi_{1}-G \phi_{2}\right\|_{1 / 2}\right) .
\end{aligned}
$$

This shows that $f$ satisfies the condition $\left(\mathrm{H}_{2}\right)$. Hence by Theorem 3.3, the mild solution of (4.2) is unique.

\section{Acknowledgments}

This research was supported by the National Natural Science Foundation of China (Grant no. 11261051), the Fundamental Research Funds for the Gansu Universities and The Project of NWNU-LKQN-11-3.

\section{References}

[1] L. Gaul, P. Klein, and S. Kemple, “Damping description involving fractional operators," Mechanical Systems and Signal Processing, vol. 5, no. 2, pp. 81-88, 1991.

[2] W. G. Glockle and T. F. Nonnenmacher, "A fractional calculus approach to self-similar protein dynamics," Biophysical Journal, vol. 68, no. 1, pp. 46-53, 1995.

[3] R. Metzler, W. Schick, H. G. Kilian, and T. F. Nonnenmacher, "Relaxation in filled polymers: a fractional calculus approach," Journal of Chemical Physics, vol. 103, no. 16, pp. 7180-7186, 1995.

[4] F. Mainardi, "Fractional calculus: some basic problems in continuum and statistical mechanics," in Fractals and Fractional Calculus in Continuum Mechanics, vol. 378 of CISM Courses and Lectures, pp. 291348, Springer, Vienna, Austria, 1997.

[5] R. Hilfer, Applications of Fractional Calculus in Physics, World Scientific, River Edge, NJ, USA, 2000.

[6] I. Podlubny, Fractional Differential Equations, vol. 198 of Mathematics in Science and Engineering, Academic Press, San Diego, Calif, USA, 1999.

[7] M. M. El-Borai, "Some probability densities and fundamental solutions of fractional evolution equations," Chaos, Solitons and Fractals, vol. 14, no. 3, pp. 433-440, 2002.

[8] M. M. El-Borai, "Semigroups and some nonlinear fractional differential equations," Applied Mathematics and Computation, vol. 149, no. 3, pp. 823-831, 2004.

[9] V. Lakshmikantham, S. Leela J, and J. Vasundhara Devi, Theory of Fractional Dynamic Systems, Cambridge Scientific, 2009.

[10] R. P. Agarwal, V. Lakshmikantham, and J. J. Nieto, "On the concept of solution for fractional differential equations with uncertainty," Nonlinear Analysis: Theory, Methods \& Applications, vol. 72, no. 6, pp. 2859-2862, 2010.

[11] Y. Zhou and F. Jiao, "Existence of mild solutions for fractional neutral evolution equations," Computers E Mathematics with Applications, vol. 59, no. 3, pp. 1063-1077, 2010.

[12] J. Wang and Y. Zhou, "A class of fractional evolution equations and optimal controls," Nonlinear Analysis: Real World Applications, vol. 12, no. 1, pp. 262-272, 2011.

[13] R.-N. Wang, T.-J. Xiao, and J. Liang, "A note on the fractional Cauchy problems with nonlocal initial conditions," Applied Mathematics Letters, vol. 24, no. 8, pp. 1435-1442, 2011.

[14] H. Ye, J. Gao, and Y. Ding, "A generalized Gronwall inequality and its application to a fractional differential equation," Journal of Mathematical Analysis and Applications, vol. 328, no. 2, pp. 1075-1081, 2007. 
[15] M. M. El-Borai, "Semigroups and some nonlinear fractional differential equations," Applied Mathematics and Computation, vol. 149, no. 3, pp. 823-831, 2004.

[16] A. A. Kilbas, H. M. Srivastava, and J. J. Trujillo, Theory and Applications of Fractional Differential Equations, vol. 204 of North-Holland Mathematics Studies, Elsevier Science B.V., Amsterdam, The Netherlands, 2006.

[17] A. Debbouche and D. Baleanu, "Controllability of fractional evolution nonlocal impulsive quasilinear delay integro-differential systems," Computers $\mathcal{E}$ Mathematics with Applications, vol. 62, no. 3, pp. 14421450, 2011.

[18] A. Ashyralyev, "A note on fractional derivatives and fractional powers of operators," Journal of Mathematical Analysis and Applications, vol. 357, no. 1, pp. 232-236, 2009.

[19] I. Podlubny, Fractional Differential Equations, vol. 198 of Mathematics in Science and Engineering, Academic Press, San Diego, Calif, USA, 1999.

[20] A. Babakhani and V. Daftardar-Gejji, "On calculus of local fractional derivatives," Journal of Mathematical Analysis and Applications, vol. 270, no. 1, pp. 66-79, 2002.

[21] M. De la Sen, "About robust stability of Caputo linear fractional dynamic systems with time delays through fixed point theory," Fixed Point Theory and Applications, vol. 2011, Article ID 867932, 2011.

[22] M. De la Sen, "Positivity and stability of the solutions of Caputo fractional linear time-invariant systems of any order with internal point delays," Abstract and Applied Analysis, vol. 2011, Article ID 161246, 25 pages, 2011.

[23] D. Baleanu, K. Diethelm, E. Scalas, and J. J. Trujillo, Fractional Calculus, vol. 3 of Series on Complexity, Nonlinearity and Chaos, World Scientific, Hackensack, NJ, USA, 2012.

[24] A. Pazy, Semigroups of Linear Operators and Applications to Partial Differential Equations, vol. 44 of Applied Mathematical Sciences, Springer, New York, NY, USA, 1983.

[25] H. Amann, "Periodic solutions of semilinear parabolic equations," in Nonlinear Analysis, pp. 1-29, Academic Press, New York, NY, USA, 1978.

[26] P. E. Sobolevskii, "Equations of parabolic type in a Banach space," American Mathematical Society Translations Series 2, vol. 49, pp. 1-62, 1966.

[27] H. Liu and J.-C. Chang, "Existence for a class of partial differential equations with nonlocal conditions," Nonlinear Analysis: Theory, Methods \& Applications, vol. 70, no. 9, pp. 3076-3083, 2009.

[28] C. C. Travis and G. F. Webb, "Existence, stability, and compactness in the $\alpha$-norm for partial functional differential equations," Transactions of the American Mathematical Society, vol. 240, pp. 129-143, 1978. 


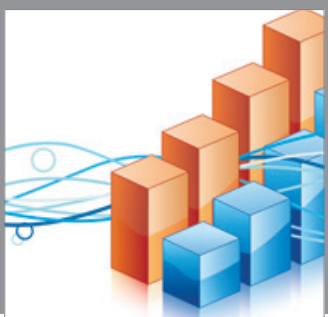

Advances in

Operations Research

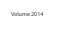

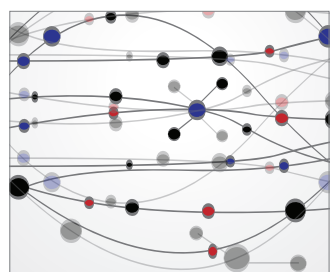

\section{The Scientific} World Journal
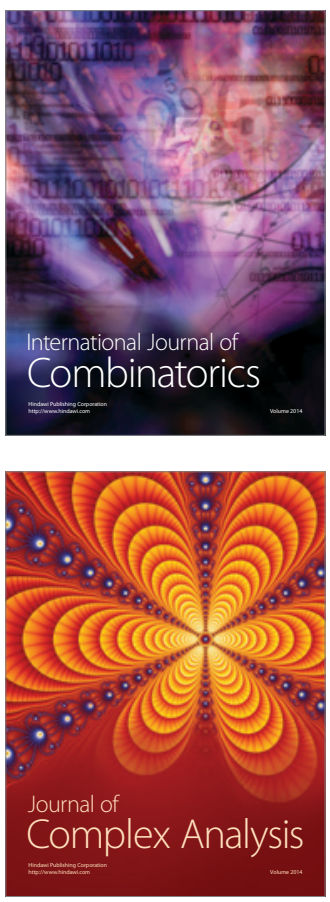

International Journal of

Mathematics and

Mathematical

Sciences
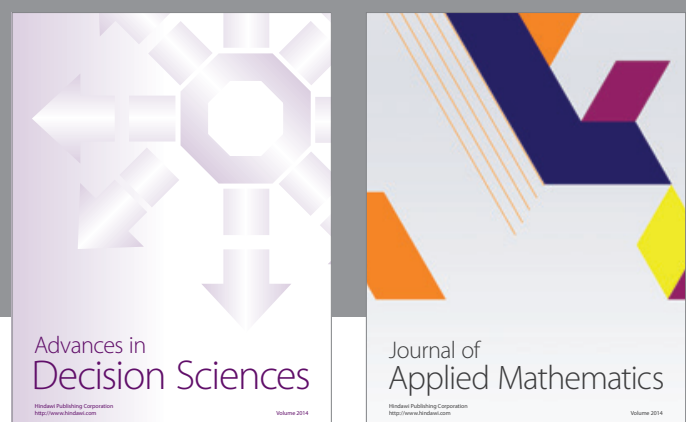

Journal of

Applied Mathematics
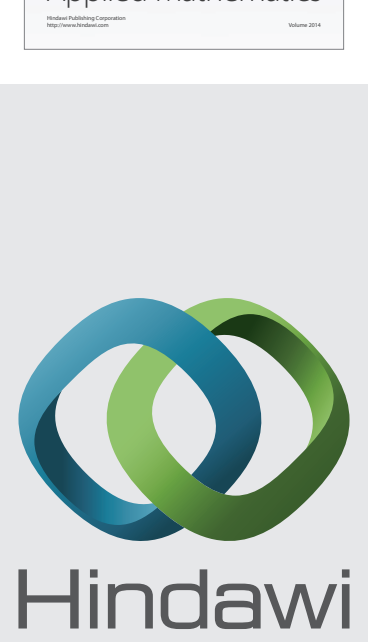

Submit your manuscripts at http://www.hindawi.com
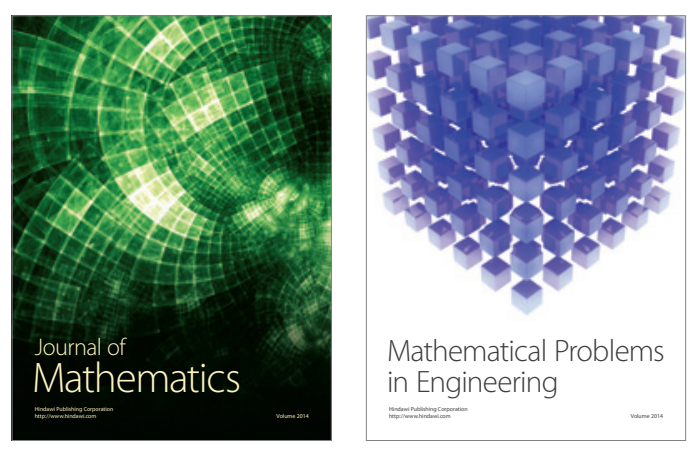

Mathematical Problems in Engineering
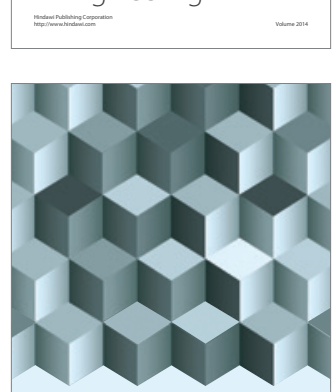

Journal of

Function Spaces
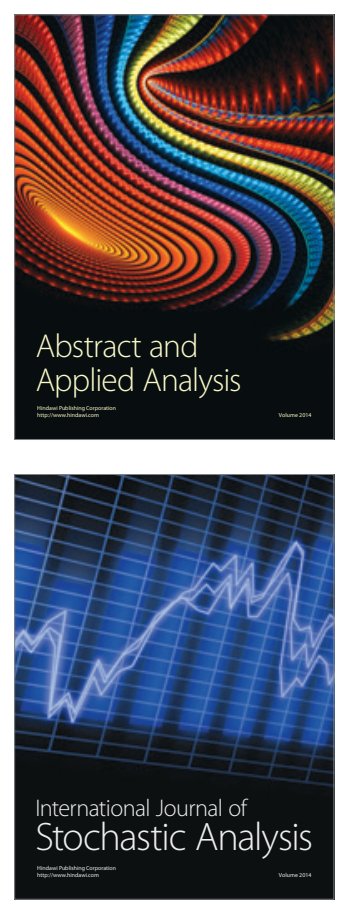

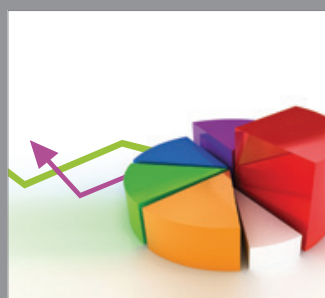

ournal of

Probability and Statistics

Promensencen
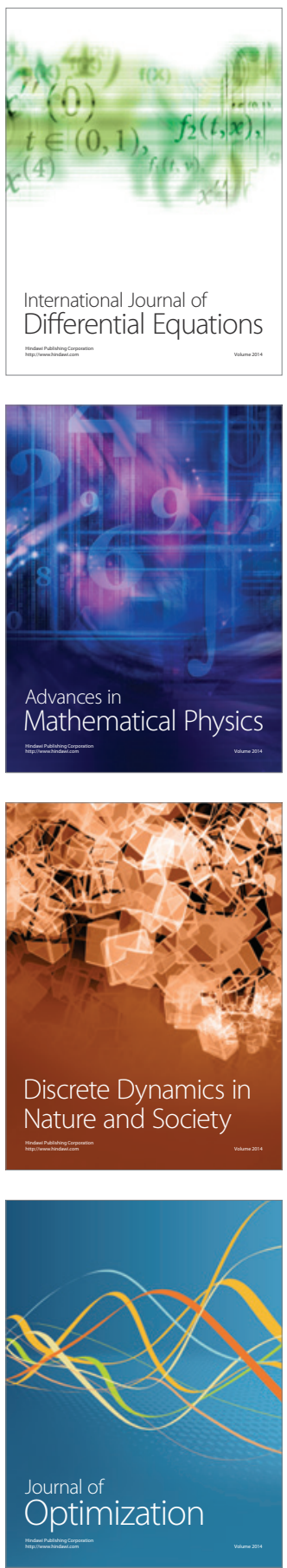